\title{
Breathe easy: Model and control of human respiration for computer animation
}

\author{
Victor B. Zordan *, Bhrigu Celly, Bill Chiu, Paul C. DiLorenzo \\ University of California, 900 University Ave. Riverside, CA 92521, USA
}

Received 15 January 2005; received in revised form 26 March 2005; accepted 29 March 2005

Available online 14 July 2005

\begin{abstract}
In this paper, we detail an anatomically inspired, physically based model of the human torso designed for the visual simulation of respiration using a mixed system of rigid and deformable parts. Motion related to breath is a signature movement of the human body and an indicator for life but it has been largely overlooked by the graphics community. A novel composition of biological components is necessary to capture the key characteristics of breathing motion visible in the human trunk because the movement is generated fundamentally through the combination of both rigid bone and soft tissue. Our approach uses a simple physically based muscle element which is used throughout to drive the motion of the ribs and diaphragm as well as in other muscles, like those of the abdomen, to produce passive resistance. In addition, we describe an implementation of a straightforward method for preserving incompressible volume in deformable bodies to use in approximating the motion of the abdomen related to breath. Through the careful construction of this anatomically based torso, control for respiration becomes the generation of periodic contraction signals for a minimal set of two muscle groups. We show the flexibility of our approach through the animation of several breathing styles using our system.
\end{abstract}

(c) 2005 Elsevier Inc. All rights reserved.

Keywords: Human simulation; Physics-based animation; Anatomical models; Animation control

\footnotetext{
* Corresponding author. Fax: +1 9517874643.

E-mail addresses: vbz@cs.ucr.edu (V.B. Zordan), bcelly@cs.ucr.edu (B. Celly), bill@cs.ucr.edu (B. Chiu), pdiloren@cs.ucr.edu (P.C. DiLorenzo).
} 


\section{Introduction}

In animation, motion, and deformation of the torso have remained stylistic and are often overly simplified or ignored entirely. To create a believable humanlike body, especially within and around the torso, and to visually bring a character to life, the movement and interplay of rigid and deformable bodies found in the trunk are invaluable. Even during rest, the trunk moves involuntary, predominantly driven by the function of the respiratory system. In this paper, we describe the design of a model which mimics the biological aspects of the torso through a straightforward physically based, anatomically inspired simulation with the specific goal of synthesizing this motion associated with human breath.

To capture the complex interactions that are seen between the variety of components in the torso related to breath, a physically based anatomical model is an obvious choice. A physical approach is superior to describing the motion procedurally because the movements of the components of the torso like the ribs and the (abdomen) gut interplay and are difficult to explain heuristically because of the mixing of deformation and rigid body motion. Movement associated with breath could be isolated during capture with data-driven skin deformation approaches $[2,33]$ but a physical torso simulation will allow fine control over the subtleties of the movement and can encapsulate a range of behaviors in a single representation that can generate novel motion immediately without the need for additional recording.

To create the desired visual effects found in the motion of breath, we describe a composite torso simulation which combines rigid-body dynamics with elastically deformable bodies shown in Fig. 1. The simulation uses spring-based muscles to estimate forces that pull and deform connected objects and estimated pressure forces to preserve the volume of the deformable components. Because the human body incorporates soft, deformable organs and muscles with (mostly) rigid bones, approaches which capture only one form of motion, deformable or rigid-body, are insufficient for the task and will lead to either computational limitations or a lack of flexibility. The use of rigid bodies and spring-based systems has appeared in numerous research and commercial arenas associated with graphics, but few have discussed the interaction of such systems $[4,28]$. Further, none to our knowledge have proposed a system of like scale which seamlessly combines such components. While we choose individual simulations of the base components that are each simple and well-understood, our design of the shape-changing torso is novel in its use and integration of these components and affords our top-level goal to faithfully recreate the complex motion associated with breath.

\section{Background}

Visual and physical simulation of synthetic anatomical muscles has been described for several applications related to modeling and animation, for example in the head and face $[19,22,30,43,47]$, the hand $[1,14]$, and for skeletal muscles $[8,26,34,40,48]$. 

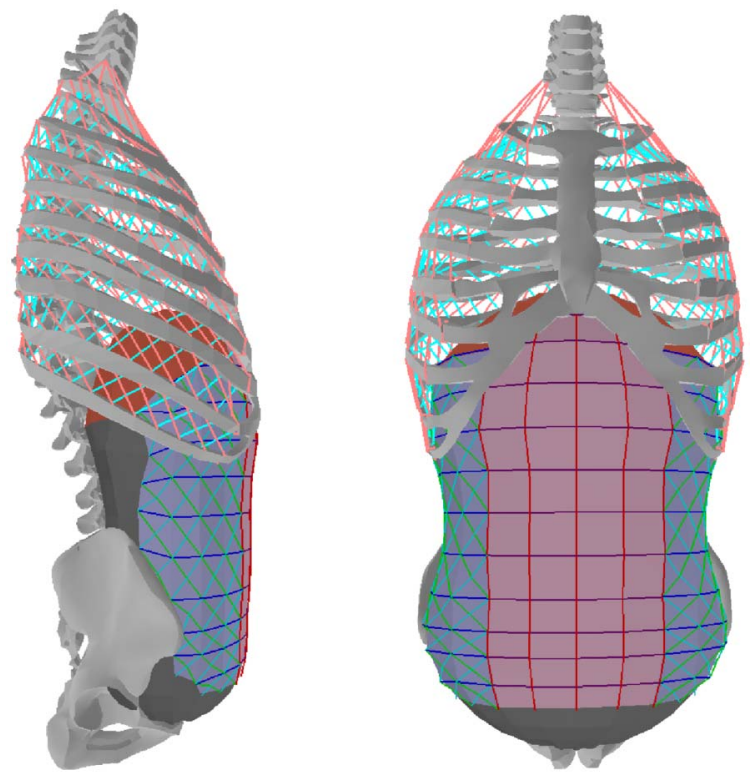

Fig. 1. Composite trunk simulation. Articulated rigid-body bones and deformable surfaces for the diaphragm and abdomen animated with springlike-muscle elements, approximately 1500 in total. Pelvis and lower and back sections of the gut are fixed in this model. Colors are assigned to groupings of springs treated uniformly in the simulation.

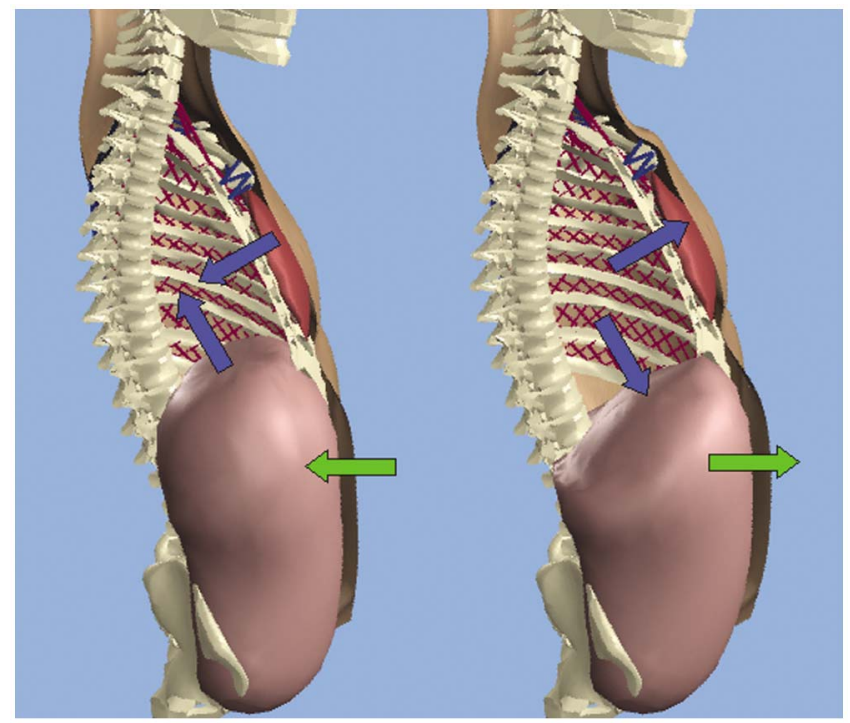

Fig. 2. Simulation shown at full inhale (right) and full exhale (left). This shows the movement of the trunk during breathing. The blue arrows show active movement of the ribcage outward and the diaphragm downward as it inhales. This results in the abdomen pushing outward (green arrow) as in response to this movement. 
Visual simulation of skeletal muscles has been approached procedurally through heuristic shape changes made in response to bone movement $[34,48]$. These examples model the change in shape of a muscle through geometric muscle bellies that stretch and deform based on length. Such procedural techniques have been adopted in the entertainment industry and used extensively for movies such as Disney's Dinosaur [11]. Physically based approaches for skeletal muscles include the work of Chen and Zeltzer [8] who use a biologically based muscle model to generate proper muscle force and Teran et al. $[40,41]$ who use a finite volume method (FVM) to create a continuous internal-tension based muscle simulation, focusing on the muscles of the arm. Both show results of deformation on the muscles systems of a single limb. In addition, Nedel and Thalmann [26] propose the use of a spring-mass system as an alternative for realtime applications. Closer to our efforts for respiration are the models of Kaye et al. [20] who animate deformable lungs for clinical applications based on a model built from CT scans and simplified cardiopulmonary mechanics and the constraint-based solver of Promayon et al. [31] which models the deformation of the abdomen during calm breath. However to the best of our knowledge, ours is the first work to investigate the animation of torso by simulating the motion of both the ribcage and gut.

Our system combines a custom deformable simulation system, that preserves volume based on pressure, with an available rigid-body dynamics solver, Open Dynamics Engine [37]. Since the pioneering work by Terzopoulos et al. [42,44] introduced the use of differential equations to animate deformation, numerous researchers have suggested techniques for interactive and multi-resolution deformable simulation, including $[6,10,16,18,25]$. In general, exact volume preservation is not guaranteed by a given deformation system, though it may afford a structurally supported volume, for example, by constructing objects using 3D tetrahedrons elements, as Müller et al. [25] demonstrate. Deformation with explicit volume preservation has been managed in fewer cases: several suggest techniques using constraint solvers and optimization [29,31,32]; Cani-Gascuel and Desbrun [5] use implicit surfaces and add a translation function to the surface displacement to account for changes in volume; and Teran et al. [40] allow for preservation through a volumetric term added to the internal tension of a muscle modeled with FVM. Also, a real-time approach is offered by Stahl et al. [38] for simulation of tissue volume with a constrained "bag of particles."

While rigid-body dynamics is well-understood and described in many texts, control for motion has been the focus of most rigid-body related papers found in the literature for computer graphics. Though no truly general solutions for control have been offered to date, most have simplified muscle activation to torque-generated actuation. Because we use direct muscle force activation in lieu of torque-driven motion, we save remarking on these many efforts for brevity. Techniques using force-based controllers for simulated behaviors are much less common, two examples being the spring-actuated controllers employed to animate flexible models for snakes and fishes $[23,46]$. To create behaviors for slithering and swimming, control systems are constructed with hand-tuned input parameters for sinusoids that move the body through coordinated forces. Follow-up work shows that optimization is useful in generating these control parameters automatically [17]. Other related 
approaches introduce alternative methods for controlling free-form deformations and mass-spring lattices $[9,12,49]$.

Data-driven methods offer alternative approaches to physically based models for creating realistic motion. Most recently, capture technologies like full-body scanners, motion-capture systems, and high-resolution digital cameras have given rise to fullbody reconstruction [3,35] and data-driven animation with deformation [7,33]. Allen and co-workers [2] also present a data-driven approach that animates muscle deformation by interpolating scans, showing results that include visually compelling muscle flexing and stretching. Captured examples undoubtedly contain considerable detail about the real world but data-driven approaches may fail to produce realistic deformation for conditions far different from those embedded in the given dataset. Thus, while these methods have been shown to produce impressive results, the use of fundamental physical models holds greater promise for general synthesis under novel situations.

\section{Respiration mechanics}

As a foundation for the remainder of this paper, we briefly introduce the functions and constituents of human breath in the torso and define the pertinent technical vocabulary. In support of our goals associated with faithful representation of anatomical breathing, we learned a great deal about the mechanics of respiration and the muscles involved from various helpful references $[15,24,36,39,45]$. The visual motion of human breath is derived from two actively moving muscle groups - the diaphragm and the intercostal muscles attached to the ribs. These two active components lead to the movement of the chest, shoulder, arms and abdomen and even, through the spine, the involuntary motion of the head associated with breath. In the ribcage, the inner and outer intercostal muscles between the ribs change the shape of the ribcage overall and drive passive deformations of many of the chest and back's muscles. The diaphragm, found at the base inside the ribcage and attached along its perimeter, works with the rib muscles to expand the lung cavity. During relaxed breath, this muscle, shaped like an inverted bowl, pushes downward on the internal organs below, creating the reciprocal motion in the abdomen wall. These processes are summarized schematically in Fig. 2. Ironically, for the sake of visual simulation, the lungs - critical to actual breath - do not affect the outward appearance of the trunk in noticeable ways during regular respiration.

Functionally, the control that drives breath is split between the two moving systems of the ribcage and diaphragm/abdomen. These parts move in a synchronized manner and do affect each other but have unique control input based on their own neural activations [24] and very different means for using the active muscles described during inhale and exhale. The outer and inner intercostals act in opposition to each other and, based on the relative position of their origin and insertion points, they allow the ribcage to open and close (respectively) on its own. In contrast, the movement of the abdomen wall surrounding the gut is indirectly driven by the pumping of the diaphragm and stores potential energy through inhalation to reset the 
diaphragm during exhalation. During inhale, the balance of surface tension and increased internal pressure caused by the downward plunging diaphragm yields the movement of the abdomen wall. As the diaphragm moves downward, the front and sides of the abdomen move outward. Upon exhalation, as the diaphragm relaxes and the pressure drops, the muscles of the abdomen release slowly, leading to a gentle return.

\section{Anatomical spring-muscle elements}

To create the anatomically based model desired, we derive a simple element that will be connected in parallel and in series to form muscles of choice. We propose controllable, spring-like muscle elements based on two fundamental assumptions about real muscles. First, a muscle can contract only after being stretched due to the forcelength-velocity relationship of muscle force production. At shorter lengths, muscles are incapable of generating force. Hence, they must be stretched first to move back to their operating length range. Zajac includes further detail about this concept in [50]. Second, muscles contain a damping component that acts to resist contraction based on the speed of shortening. The latter is supported by the findings of Hill, as cited by Chen and Zeltzer $[8,13]$. The former (which is easily understood by considering the likeness muscles to rubberbands) implies that muscle forces must only act in tension and have negligible (zero) force in compression. Functionally, expansion must be triggered by opposing contraction of the form found in the muscle pairs of the intercostals in the ribcage, or by some other external influence, like the pressure difference which compels the return of the diaphragm during exhale. Thus, only after being stretched can the diaphragm muscle again contract. And, important for controlled breathing, after muscles are stretched and in tension, they damp and resist contractive movement to form a slow passive release to rest as seen in the abdomen's relaxation during exhale.

Given these constraints, we propose the following simple calculation for passive elemental muscle forces based on the length of the element, $\ell$, and its derivative $\dot{\ell}$ :

$$
F_{m}=\min \left[-k\left(\ell-\ell_{\mathrm{o}}\right)-b \dot{\ell}, 0\right],
$$

where $k$ and $b$ are the stiffness and damping gains of the element and $\ell_{\mathrm{o}}$ is its rest length. This piecewise-linear function acts like a linear spring and damper unless the value computed is positive in which case it is set to zero, invalidated by our non-compressive constraint. Neff and Fiume point out that two linear springs can equivalently replace the single [27], and, as such, to create actuation in the spring elements while maintain a separate passive muscle characteristic, we modify the force calculation to

$$
F_{m}=\min \left[-\alpha k_{\mathrm{ac}}\left(\ell-r \ell_{\mathrm{o}}\right)-k_{\mathrm{pe}}\left(\ell-\ell_{\mathrm{o}}\right)-b \dot{\ell}, 0\right],
$$

where $k_{\mathrm{ac}}$ and $k_{\mathrm{pe}}$ are the active contraction and passive elastic gain values. The contraction is controlled based on $r$, the desired contraction ratio for $\ell_{\mathrm{o}}$, and the normal- 

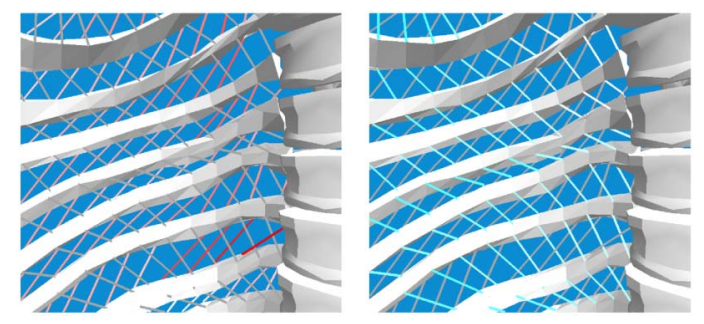

Fig. 3. Intercostal spring elements. The outer and inner intercostals contract during inhale and exhale respectively. Their function is derived from their connection points which align the muscles along the circumference of the ribcage at close to right angles to each other.

ized actuation level, $\alpha$. While we use linear parts, we model our terms after the more sophisticated Hill-type muscle models described by Zajac [50].

Our muscle-element model is both simple to compute and intuitive to tune, especially related to the passive effects and controlled damping of a muscle, as mentioned an important behavior in breathing. Note, Eq. (2) allows a 'virtually' positive damping component, that may be included as long as the net force of the muscle remains negative. This prevents the muscle from contracting too quickly (actively or passively), while helping to reduce large contractile forces before they are applied. And, by maintaining a unique component for passive elasticity, the muscles' passive characteristics may be determined separately, for example through simpler (passive) experimentation. Then, to tune active motion, the properly tuned passive and damping components provide a good starting point for the actuation tuning associated with the specific desired, controlled behavior.

As Fig. 1 shows, we model whole muscles as sets, or groups, of individual muscle elements which act on neighboring muscles and bones based on their local attachments points. Attachment points mimic the continuous origin and insertion points of the muscles in the human body with discrete sparsely sampled insertions of the elements as in Fig. 3.

\section{Rigid-body components}

Rigid-body simulation for skeletal motion has saturated the field and versions appear regularly in films and games, but our use of rigid bodies is quite different than many reported. Most often, the trunk is broken into one to three, possibly five, rigid sections - splitting along the spine and, at times, incorporating clavicle motion in the shoulders. To create a faithful simulation of breathing motion, the individual movement of the ribs is required and our simulation of the ribcage includes the rigid-body segments for the spine plus 10 moving ribs per side, and a separate body for the sternum where dynamic parameters are estimated based on the geometric models' volume and uniform density. To create many of the animations for this work, we 
also include additional rigid-body arms with three rigid sections for the combined shoulder/clavicle and upper and lower arms.

To afford the desired range of motion, we use mixed forms of connections, based on the amount of 'play' desired. For true bone-to-bone connections, we use ball joints, for example connecting the ribs to the spine and the ball joint of the shoulder at the top of the upper arm. We use a structurally stable configuration of spring elements to mimic more flexible connections, for example attaching the front of the ribs to the sternum. This connection in the human body is made with flexible cartilage. For the sake of simulation complexity, we opt to make this simple approximation of the cartilage and allow the springs to incorporate the small amount of play required for a reasonable range of motion. Incidentally, with fixed ball joints connecting the ribs to the sternum, the rigid-body simulation becomes overly constrained and an unsuitable range of motion results.

Unlike many approaches for driving rigid-body motion with joint control torques, we exclusively use forces, computed from our spring-muscles elements, to drive the movement of the rigid components. Relatively few animation works describe using such techniques, even though the general approach more closely matches the motion induced in real human. And, with the use of realistic insertion points and valid, noncompressive muscle forces (that pull, but not push) this technique helps to constrain the possible movements and yields an easily controlled rigid-body system. For example, rather than deciphering the complex torque input required to move each rib in a proper oscillation pattern for steady-state breath, the interleaved contraction of the inner and outer intercostal muscles leads to valid, stable movement without the need for extraneous collision detection between the ribs or any form of high-level feedback or knowledge within the controller.

Through experimentation, we found that a small amount of joint friction produces pleasing results. Initially, we made the assumption that the joints were frictionless - the shape of bone interfaces and the slippery cartilage between work to minimize friction and support this assumption. However, after several attempts to discern the cause of the proper sway of the spine in conjunction with breath, we added rotational friction of the form:

$$
\tau_{\text {fric }}=-\mu \dot{\theta}
$$

to the connective joints between the ribs and the spine. The torque, $\tau$, is applied at the joints to the spine and the ribs, based on each joint's angular velocity, $\dot{\theta}$, and friction coefficient $\mu$. Through these friction-based torques, as the ribs move, the spine moves. For example during a deep inhale, as the ribs are pulled upward, the spine moves backward in a visually pleasing manner.

\section{Soft-body components}

We synthesize the motion of the abdomen wall by modeling the gut as a deformable, incompressible volume. In the human gut, the intestines and other internal organs lay inside the thorax liner and are flexibly displaced as the diaphragm pushes 
down during inhalation, subsequently pushing on the muscles of the abdomen. We abstract away the internal organs and consider only their effect on the sealed liner, treating the gut as a closed system that encases the inner organs. The assumption here is that the bulk of the gut's volume is incompressible and that the effects of the local structure, the intestines for example, are negligible compared to this incompressibility. Supporting this assumption, we combine a simple deformable surface model with a straightforward volume-preserving routine.

\subsection{Deformation}

During inhale, the change in shape of the abdomen wall is dictated largely by the passive resistance of muscle elements associated with the transversus, the inner and external obliques, and the rectus. We model this layer of muscles along with the gut liner using strands of spring-muscle elements that follow the nominal directions of the actual muscles' fibers wrapping around the abdomen (see Fig. 1). A synthetic diaphragm lies at the top of the gut 'body' and changes shape based on its own contraction as well as the internal pressure forces of the gut. A fixed backside and bottom are added to the 'gut-body' system to complete the sealed volume. For deformation, the system computes the associated spring-muscle forces from the abdomen and diaphragm muscle groups and applies them to a distribution of point masses placed at the spring intersections, updating the masses using simple explicit Euler integration with equal mass values for each point based on an estimated mass of the abdomen wall (est. as $8 \mathrm{~kg}$ total.) External forces are added determined both based on the neighboring spring-muscle elements and the pressure forces. Obviously, higher-order explicit and implicit integration methods would lead to more stable and faster simulation but we found Euler integration satisfactory for our purposes.

\subsection{Volume preservation}

With volume preservation, the gut-body simulation deforms through a balance of surface tension and internal pressure forces, emulating the physical nature of the human gut as it moves during breath. According to Mines, Hooke's law applies to many compliant (biological) structures over their physiological range and upholds that volume varies linearly with pressure [24]. We use this relationship to compute the pressure based on the original volume of the body, $V_{\mathrm{o}}$ and current volume, $V$ :

$$
P=\kappa\left(\frac{V_{\mathrm{o}}}{V}-1\right)
$$

where $\kappa$, or the bulk "volumetric" modulus (naming convention based on the like term described by Teran et al. [40]), controls the quasi-incompressibility. Volume estimation is approximated from the sum of a set of pyramidal volumes defined between the mass center of the body and each face on its surface, similar to [5]. A pictorial representation can be seen in the top image of Fig. 4. For our periodic breathing examples, we found $\kappa=200$ to be satisfactory for the gut calculations. 


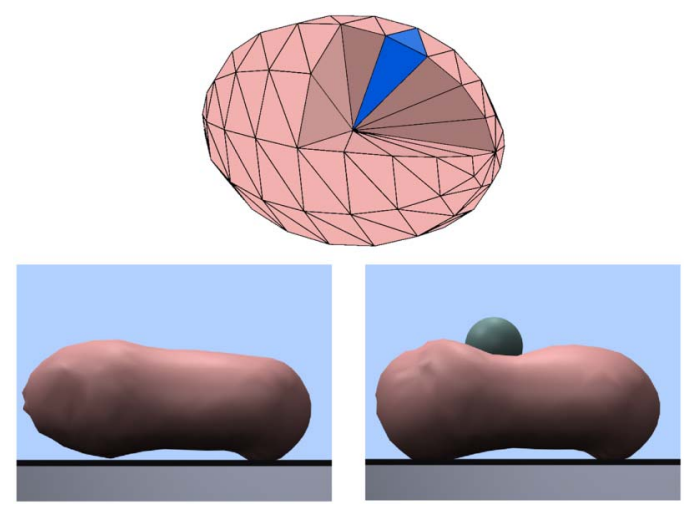

Fig. 4. Volume calculation cutaway (top). To compute the volume of the triangulated mesh, each triangle of the mesh becomes a tetrahedran using a single internal point. The summation of the volume of each tetrahedran is the total volume of the mesh. Gut deformation after a heavy impact (bottom). The volume in this animation showed an error less than $0.5 \%$ and revealed a tolerable error less than $2 \%$ during our results for breathing.

To approximate the pressure forces based on the deformation simulation, we triangulate the gut-body surface, compute the area and normal of each triangle, and distribute pressure force evenly among the constituent vertices. Thus, for each mass point $j$, a pressure force equivalent to

$$
F_{i j}=\max \left[0, \frac{P A_{i}}{3} \mathbf{n}_{i}\right]
$$

is applied for each neighboring face $i$. Here, zero pressure force replaces a negative force under the assumption that the gut is subjected to negligible (atmospheric) pressure from the outside and nothing acts to pull the wall inward. A demonstrative example of this can be seen in the bottom image of Fig. 4 where we drop a lead ball on the gut body.

Muscles of the abdomen are modeled with muscle elements connected in series along the surface of the gut based on their direction. Three set of springs along each side of the gut running upward, downward, and horizontal, match the general directions of the layered muscles found in the obliques and transversus. The rectus muscles, acting vertically, also connect the abdomen (gut-body) to the ribcage. Each of the muscle groups is assigned its own gain and damping values.

\section{Muscle activation for breath control}

Actuating several hundreds of muscle elements, even the simple ones proposed here, to create a single coordinated movement in a desired manner requires a practical means of control. We manage a large portion of this complexity through careful modeling and the use of low-level controllers that compute forces based on local 
conditions. Through intuitive user handles, the remainder of control comes from the tuning of the groups' collective muscle-element spring parameters.

We control activation for muscle groups by changing the relative contraction value, $r$, as a time-varying input parameter and chose to establish $\alpha$ as a binary switch which moved between on and off at appropriate times, with timing of each based on the frequency of the desired breath. With experimentation, we found this approach to provide intuitive user-handles for muscle actuation. We select contraction ratios in accordance with modeled and measured contraction ranges of actual human muscles. For comparison, based on a nice summary of such research, Klute et al. [21], selected a desired contraction range of 0.7-1.2 for their artificial muscles. Mines implies that the intercostals and diaphragm are controlled uniquely based on their neural pathways' differing connection points with the spine [24] and, as such, we supply two unique patterns for the rib and diaphragm contraction input signals. While the true nature of such activation remains a mystery according to our background search, such inputs have been proposed previously to control animation of physical muscle simulations: Chen and Zeltzer [8] used handcrafted curves as input for activation; Tu and Terzopoulos [46] used sinusoids; and Teran et al. [40] offer a hand keyframed "animator-friendly" ratio based on maximal contractive force. Through iterative adjustments, we found the span from 60 to 80 percent of the rest contraction length to be useful for active contraction of the diaphragm during a range of breaths and for the rectus during forced exhale.

For normal, steady-state breathing, periodic contraction signals allows the highlevel control of the breath frequency directly, usually $13-17$ breaths per minute $(\mathrm{b} / \mathrm{m})$ in the average human [36]. We experimented with the use of both smooth and abrupt changes for the periodic contraction using simple sinusoid and step functions. We found that, for the diaphragm, often referred to as a pump or a plunger, a step function created the desired response. For the intercostals, out-of-phase sine curves lead to visually pleasing, smooth oscillations for the ribcage motion. From the actuation inputs, the control system determines the individual spring forces. Thus, upon inhale, the outer intercostals contract with a smoothly dropping $r$ leading to the opening forces on the ribs. Meanwhile, as seen in Fig. 2, the diaphragm plunges down pushing on the gut. At the time of exhale, the contraction in the outer intercostals is slowly released as the inner intercostals begin to contract and the diaphragm releases to allow its return under the internal pressure forces created by the stretched abdomen wall. And so on, the cycle continues.

\section{Secondary components and skin}

Once the primary moving parts of the torso simulation are in place, we layer on secondary motion for the shoulders, chest, and arms following one-way coupling as described by O'Brien et al. [28]. A rigid chain of segments for the bones of the shoulder and arms link to the trunk between the sternum and collarbone on either side and move under the influence of several spring muscle groups attached to the spine, 
ribs, and sternum at anatomically close insertion points. Two pairs of deformable muscle bodies are added for the pectorals and the bulk of the shoulder (mostly, the trapezius, and deltiods.) These are simulated as volume-preserving bodies and attached with a number of strategically placed, zero-length springs. Their shape is dictated both by the movement of the underlying parts and the volume constraints described in Section 6. Due to its unusual shape, we found it necessary to support the surface of the shoulder body with a small number of internal spring supports. Although we believe this could be avoided by splitting the aggregate shoulder into its constituent muscles, it seemed sufficient for the small amount of movement anticipated of the shoulder muscles during breathing behaviors.

We generate a skin surface based on trajectories of trace vertices that are recorded during simulation and used as control points for a NURBS surface computed through Alias' Maya via MEL-scripting. We add a small percentage offset to account for the layers between the muscle/bone layer and the skin, (non-uniform fat layers remains an interesting area for future work). In practice, once the simulation is complete, the resulting data may be displayed in any number of ways as represented by the figures in this paper, and the proper display should be based on the application. The skin shown in our results only represents one simple, but illustrative example. A progression showing the layering of these secondary elements and the final skin appears in Fig. 5.

\section{Design remarks and implementation}

There are many geometric parameters and engineering decisions embedded in the torso model and the breathing simulation. Before describing specific parameters used, we highlight a few of the straightforward design principles that helped direct our choices, listed in prioritized order from highest to lowest:

- Where possible and pertinent, follow the anatomical form of the human body, both in its physical makeup and in the local modes it uses to accomplish a given task.

- Consider the important tradeoff between the complexity of the model of a given component and the limitations the model imposes on the control and range of movements afforded by the proposed model in selecting individual components.

- Without compromising the anatomical correctness or adding difficulty to the modeling and control of a given component, reduce the resolution and simplify the structure to support both simulation stability and efficient computation.

These guidelines lead us to the design of the torso model as described. Tables 1-3 list mass, stiffness, control, and other statistics that comprise the system implemented based on this model. Our coarse but anatomically similar skeleton model, downloaded from 3D Cafe (www.3Dcafe.com,) includes 22 rigid body segments for the rigid-body torso approximation (plus an additional six for the arms.) We believe this to be a minimal number of segments possible for the modeling of 

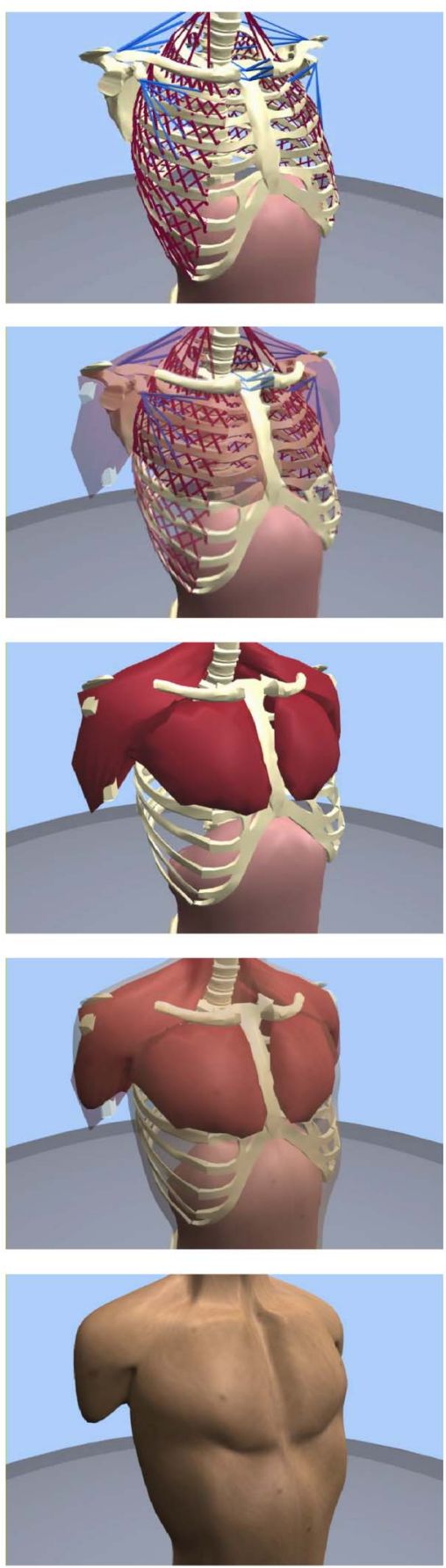

Fig. 5. Layers of the torso model. 
Table 1

Muscle group stats

\begin{tabular}{lcccc}
\hline Muscle group & No. of springs & $k_{\mathrm{ac}}$ & $k_{\mathrm{pe}}$ & $b$ \\
\hline Intercostals & 516 & 20 & - & 1.0 \\
Diaphragm & 464 & 4 & 2.1 & 0.1 \\
Rectus & 85 & 15 & 1.5 & 0.1 \\
Transversus/obliques & 350 & - & 10 & 0.1 \\
Misc. shoulder & 52 & - & & 1.0 \\
\hline
\end{tabular}

Table 2

Body masses and attachment stats

\begin{tabular}{llllll}
\hline Body & Mass $(\mathrm{kg})$ & Inboard body & Connect type & $k_{\mathrm{pe}}$ & $b$ \\
\hline Spine/head & 10.5 & Ground & Spring & 5000 & 450 \\
Ribs & $\sim 0.7 \mathrm{per}$ & Spine & Ball joint & - & $\mu=0.3$ \\
Sternum & 2.0 & Ribs & Spring & 100 & 1 \\
Gut & 8.0 & Ribs & Spring & 40 & 1 \\
\hline
\end{tabular}

Table 3

Control parameters

\begin{tabular}{lllll}
\hline Breath style & Frequency $(\mathrm{b} / \mathrm{m})$ & Diaphragm $r$ & Intercostals $r(t)$ & Rectus $r$ \\
\hline Casual & 15 & 0.85 & $0.92 \pm 0.08$ & - \\
Slow/deep & 12 & 0.80 & $0.80 \pm 0.20$ & - \\
Panting & 60 & 0.80 & $0.88 \pm 0.12$ & - \\
Forced exhale & - & 0.80 & $0.80 \pm 0.20$ & 0.5 \\
\hline
\end{tabular}

the ribcage. The geometric model of the gut was created by hand, to fit the rigidbody skeleton.

To form muscles, we used both commercial software (Maya) and specially handcrafted procedural approaches to semi-automatically derive the springs and their groupings based on the geometric models. To isolate muscle regions, different shaders were assigned to the geometry in Maya and grouped into simple output files. For overlapping muscles, for example in the abdomen, we use rules like "group all 'mostly vertical' edges near the front" to find the (rectus) muscle group and so on. The springs of the diaphragm, were purposefully generated at a higher resolution to afford the desired curvature and flexibility found in the real muscle. Although a small amount of deformable motion is visible in the lower back of humans while breathing, we chose to ignore this motion and did not simulate the edges near the backside or bottom of the gut-body, instead using their original fixed location to compute proper volume. Also, in general whenever possible, the springs follow the primary directions of the muscles they model, but some concessions were made to help manage the sheer magnitude of springs appearing in the constituent components. 


\section{Results}

We investigated a number of examples related to different breathing styles at different frequencies as well as a non-periodic forced exhale created by actuating the abdomen muscles:

Calm breath. Casual, involuntary breath of healthy individuals which includes a considerable amount of abdomen motion due to the important contribution of the diaphragm. This neutral breath encapsulates the most comfortable and energyefficient sustainable respiration.

Slow deep breath. Also called 'deep belly' breathing in yoga, this movement reaches the full range of the respiratory system in the abdomen, from full inhale with maximum air capacity to the greatest expulsion. This breath is used as exercise and to maintain health, especially for the abdomen, because it forces intense stretching and full contraction.

Panting breathing. Opposite of deep 'belly' breathing, the high frequency pulsing of panting breath yields small rapid inhales and exhales where most of the motion is seen in the chest and upper torso. Such 'shoulder' breathing commonly associated with nervousness, can lead to undue stress in the overworked muscles at the top of the ribcage.

Forced exhale. In addition to periodic breathing styles, a hard forced exhale can be used to clear the lungs or its passageways. During a forced exhale, the rectus acts to pull the lower ribcage downward and inward as it collapses the abdomen deeply and quickly.

The parameter inputs required for the simulation of these breath styles are summarized in Table 3. With slight variation, we found that fixing the gains and modifying the activation inputs parameters alone gave way to visually compelling, easyto-tune motion. Blank values can be assumed to be zero, implying negligible input, for example we ignore the passive effects of the intercostals in our ribcage in lieu of easier tuning. Also, the rectus is only active during the forced exhale and has a zero $k_{\mathrm{ac}}$ otherwise.

We present several graphical results that help reveal specific details about our anatomical model. Fig. 6 shows the lung-cavity volume over time. While this value is not

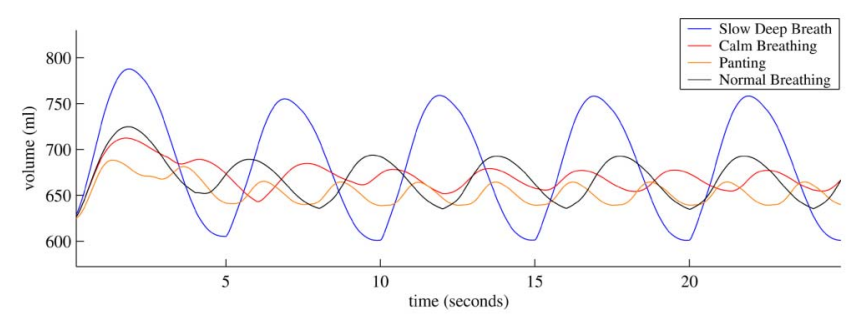

Fig. 6. Volume for various synthesized breath styles. The shown tidal (peak-to-peak) volumes computed for our simulation's lung cavity fall with in the realistic human range, with the normal breathing (black) falling almost perfectly on the $500 \mathrm{~mL}$ average quoted in the texts. Computed post-mortem, this reveals a strong correspondence between our results and that of real human motion. 


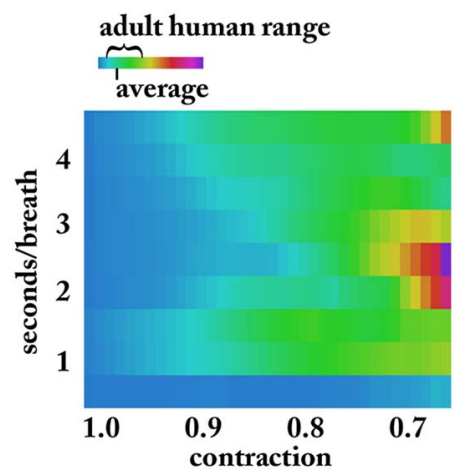

Fig. 7. Breath space. This image is the result of over 300 runs of the simulation system showing the tidal volumes for steady-state breathing associated with the space of possibilities ranging from little to large contraction inputs (along the horizontal axis) and the increasing frequencies from 0.5 seconds per breath (panting breath) to 4.5 seconds per breath. The valid adult range and normal average for the tidal volumes are indicated in the color spectrum above. The inefficiency of panting breath at drawing air into the lungs is also nicely displayed in this visual representation.

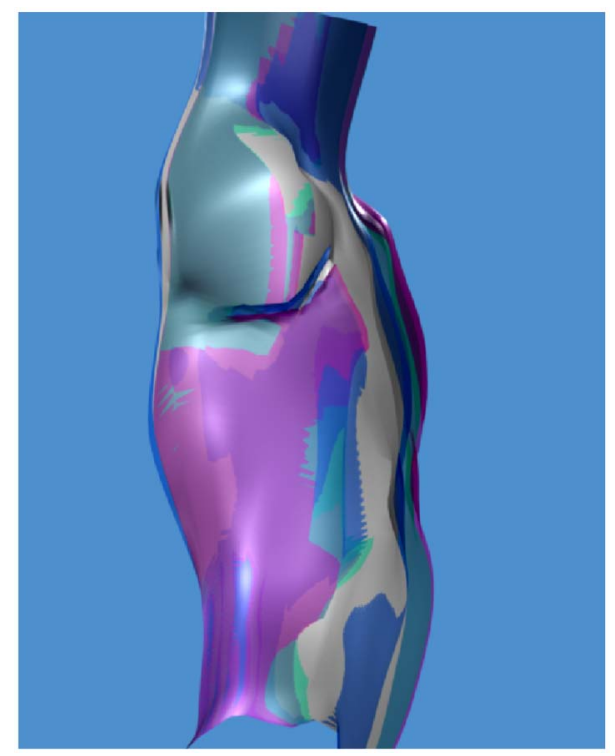

Fig. 8. Surface change. This figure overlays four cutaway snapshots of the skin surface during normal breathing to highlight the various ways in which the animated torso changes in subtle ways. While the obvious rise and fall of the chest and stomach are seen (at the right), the volume changes in the gut, the movement in the neck, and the shape change of the ribcage overall reveal a complex time-varying surface that would be difficult to generate in a purely procedural or keyframe system, supporting the use of a physically based system. 


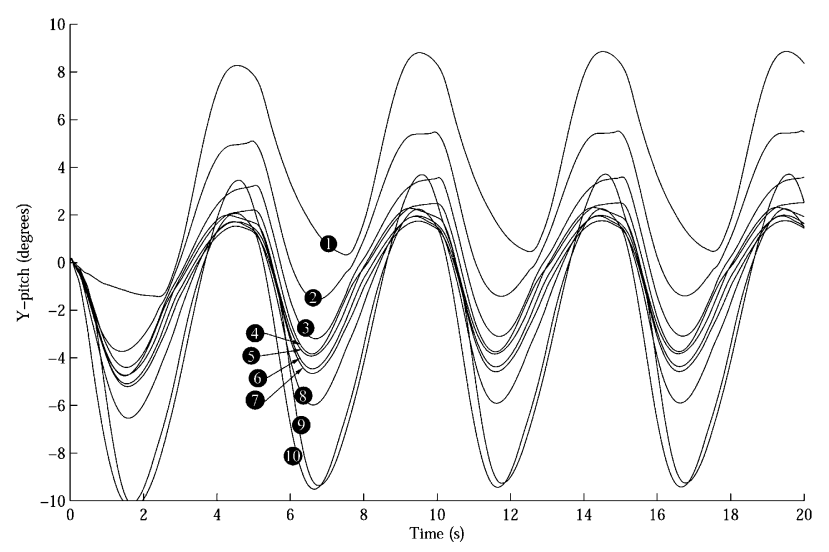

Fig. 9. $Y$-rotation for the upper righthand ribs for normal simulated breathing. Labels, 1-10, are consistent with the naming convention from standard anatomical texts. Small variations and offsets resulting in the simulation add subtleties and physical realism in the corresponding motion.

directly used in the simulation in any way, it shows that our torso is breathing in a manner close to that of a healthy average human as our computed lung volumes are comparable to those seen in the real world counterpart. A second visualization of the tidal volume for the lungs represents a space of breathing behaviors in Fig. 7, shown in comparison to human norms and ranges. Another interesting area for future work includes investigation of controlling this volume with respect to other behaviors, for example coughing or for sound or voice simulation. However, for behaviors such as these, explicit modeling of the lungs is likely to be necessary since they include air passing in and out of the larynx and trachea as a direct factor in the behavior's characteristics.

Figs. 8 and 9 reveal some of the subtleties of the simulated motion. Fig. 9 plots the ribs rotation over time. While all of the intercostals attaching these ribs receive synchronously the same control inputs, the output motion reveals delayed progression and varying extents down the ordering of the ribs. This pattern would be more difficult to generate with direct procedural animation (than with the basic sine curves used to control our ribcage). Fig. 8 shows the highly non-uniform shape changes in the skin that result from the combination of the various rigid and soft components. While it is argueable that not all of these changes directly match effects seen in a real human torso during breath, we claim that the complexity (and details) which come out of our physical model would not be easy to generate by other means and that the overall affect adds realism, in part because of the model's biological groundings.

\section{Discussion and conclusion}

While our results in this paper focus on breath, this work represents the first steps in the longer term goals of bringing a realistic human torso to life. While currently limitations include a biased effort applied to the front of the trunk to expose breath 
related effects, more general movements using this torso simulation are underway. As reported, the simulation has the mobility of a static, seated person leaning back against a low-back chair. One obvious way to build generality would be to add additional rigid-body articulation in the spine, neck, and shoulders as well as allow free movement of the torso in space. Alternatively, to use this static torso for characters "on the run," our system could generate offsets which are applied to cause shape deformations in a torso moving in freespace. In this fashion, the described system can (immediately) be employed to work in conjunction with gross pose animation in various scenarios.

We do not claim that the simulation techniques used to generate the animated torso described are themselves particularly sophisticated or efficient. We made selections for these simulation 'building blocks' both based on availability and ease of implementation. Instead, our contributions lie in our methodology and premiere investigations related to the novel application of a mixed rigid and soft simulated torso for animation as well as a focus on breath control for that system. More sophisticated simulations would still likely require the use of a mixed composition system to account for the wide range of materials that contribute to the motion of the torso. And, considering the anatomy, the problem of control for any physically based torso model will require activation of the muscles of the ribcage and the diaphragm for breathing. Computationally, without graphics or secondary elements, the torso simulation runs $60 \times$ slower than real-time (simulation time vs. actual time) on a $3.2 \mathrm{GHz}$ Athlon processor. Better computation methods would undoubtedly speed this up and we believe an efficient representation would run interactively on a modern processor.

In terms of generality across characters, while we focus exclusively on the human, the breathing styles of primates and other mammals as well as the many humanlike imaginary characters made possible by computer graphics will share key characteristics that can be managed with the same or similar approaches to the motion synthesis described here. In this context, we present both fundamental insights and design suggestions related to the implementation of an anatomically inspired trunk for any of many possible characters, largely independent of the model or simulation technique chosen.

We hope that this work will entice other researchers to consider the modeling of the human body from the inside out, based on its anatomical form. Though our model includes a fair amount of simplification, once the anatomy was modeled the desired behavior became easy to describe and manageable to control. We believe our results support the notion that, while in evolution form follows function, in the synthesis of virtual humans, the sought-for form has already crystallized and by mimicking it, humanlike function can emerge from simple mathematical models and proper excitation.

\section{Acknowledgment}

Special thanks to the reviewers who helped improve this work and its presentation, and for the support and software of Russell Smith et al. at ODE. 


\section{Appendix A. Supplementary data}

Supplementary data associated with this article can be found, in the online version, at doi:10.1016/j.gmod.2005.03.005.

\section{References}

[1] I. Albrecht, J. Haber, H. Seidel, Construction and animation of anatomically based human hand models, in: Eurographics/ACM SIGGRAPH Symposium on Computer Animation, 2003, pp. 98109.

[2] B. Allen, B. Curless, Z. Popović, Articulated body deformation from range scan data, ACM Trans. Graph. 21 (3) (2002) 612-619.

[3] B. Allen, B. Curless, Z. Popović, The space of human body shapes: reconstruction and parameterization from range scans, ACM Trans. Graph. 22 (3) (2003) 587-594.

[4] D. Baraff, A. Witkin, Partitioned Dynamics. Technical Report CMU-RI-TR-97-33, Carnegie Mellon University, 1997.

[5] M. Cani-Gascuel, M. Desbrun, Animation of deformable models using implicit surfaces, IEEE Trans. Vis. Comput. Graph. 3 (1) (1997) 39-50.

[6] S. Capell, S. Green, B. Curless, T. Duchamp, Z. Popović, A multiresolution framework for dynamic deformations. Eurographics/ACM SIGGRAPH Symposium on Computer Animation, 2002, pp. 4148.

[7] J. Carranza, C. Theobalt, M.A. Magnor, H. Seidel, Free-viewpoint video of human actors, ACM Trans. Graph. 22 (3) (2003) 569-577.

[8] D.T. Chen, D. Zeltzer, Pump it up: computer animation of a biomechanically based model of muscle using the finite element method, in: Proceedings of SIGGRAPH 92, 1992, pp. 89-98.

[9] J. Christensen, J. Marks, J.T. Ngo, Automatic motion synthesis for 3D mass-spring models, Vis. Comput. 13 (1) (1997) 20-28.

[10] G. Debunne, M. Desbrun, M. Cani, A.H. Barr, Dynamic real-time deformations using space and time adaptive sampling, in: Proceedings of SIGGRAPH 2001, 2001, pp. 31-36.

[11] Disney's Dinosaur, Disney Studios 2000. Disney Enterprises, Inc..

[12] P. Faloutsos, M. van de Panne, D. Terzopoulos, Dynamic free-form deformations for animation synthesis, IEEE Trans. Vis. Comput. Graph. 3 (3) (1997) 201-214.

[13] H. Gasser, A. Hill, The dynamics of muscular contraction, R. Soc. London Proc. 96 (1924) 393-437.

[14] J. Gourret, N. Magnenat-Thalmann, D. Thalmann, Simulation of object and human skin deformations in a grasping task, in: Proceedings of SIGGRAPH 89, 1989, pp. 21-30.

[15] H. Gray, Gray's anatomy, in: T. Pick, R. Howden (Eds.), Portland House, 1977.

[16] E. Grinspun, P. Krysl, P. Schroder, Charms: a simple framework for adaptive simulation, Proc. SIGGRAPH 2002 (2002) 281-290.

[17] R. Grzeszczuk, D. Terzopoulos, Automated learning of muscle-actuated locomotion through control abstraction, in: Proceedings of SIGGRAPH 95, 1995, pp. 63-70.

[18] D.L. James, D.K. Pai, Artdefo-accurate real time deformable objects, in: Proceedings of SIGGRAPH 99, 1999, pp. 65-72.

[19] K. Kähler, J. Haber, H. Yamauchi, H. Seidel, Head shop: generating animated head models with anatomical structure, in: Eurographics/ACM SIGGRAPH Symposium on Computer Animation, 2002, pp. 55-64.

[20] J. Kaye, D.N. Metaxas, F.P.Primiano, Jr, A 3D virtual environment for modeling mechanical cardiopulmonary interactions, in: CVRMed, 1997, pp. 389-398.

[21] G. Klute, J. Czerniecki, B. Hannaford, Artificial muscles: actuators for biorobotic systems, in: International Journal of Robotics Research, 2002, pp. 295-309.

[22] Y. Lee, D. Terzopoulos, K. Walters, Realistic modeling for facial animation, Proceedings of SIGGRAPH '95, 1995, pp. 55-62. 
[23] G.S.P. Miller, The motion dynamics of snakes and worms, in: Proceedings of SIGGRAPH 88, 1988, pp. $169-178$.

[24] A.H. Mines, Respiratory Physiology, Raven Press, New york, 1993.

[25] M. Müller, J. Dorsey, L. McMillan, R. Jagnow, B. Cutler, Stable real-time deformations, in: Eurographics/ACM SIGGRAPH Symposium on Computer Animation, July 2002, 49-54.

[26] L.P. Nedel, D. Thalmann, Real time muscle deformations using mass-spring systems, in Computer Graphics International 1998, June 1998.

[27] M. Neff, E. Fiume, Modeling tension and relaxation for computer animation, in: Eurographics/ACM SIGGRAPH Symposium on Computer Animation, 2002, pp. 81-88.

[28] J.F. O'Brien, V.B. Zordan, J.K. Hodgins, Combining active and passive simulations for secondary motion, in: IEEE: Computer Graphics and Applications, July 2000, pp. 86-96.

[29] J.C. Platt, A.H. Barr, Constraint methods for flexible models, in: Proceedings of SIGGRAPH 88 , 1988, pp. 279-288.

[30] S. Platt, N. Badler, Animating facial expression, Comput. Graph. (1981) 245-252.

[31] E. Promayon, P. Baconnier, C. Puech, Physically-based model for simulating the human trunk respiration movements, in: Lecture Notes in Computer Science, 1205:379-388, 1997. Springer Verlag CVRMED II-MRCAS III first joint conference.

[32] A. Rappaport, A. Sheffer, M. Bercovier, Volume-preserving free-form solids, IEEE Trans. Vis. Comput. Graph. 2 (1) (1996) 19-27.

[33] P. Sand, L. McMillan, J. Popović, Continuous capture of skin deformation, ACM Trans. Graph. 22 (3) (2003) 578-586.

[34] F. Scheepers, R.E. Parent, W.E. Carlson, S.F. May, Anatomy-based modeling of the human musculature, Proc. SIGGRAPH 1997 (1997) 163-172.

[35] H. Seo, N. Magnenat-Thalmann, An automatic modeling of human bodies from sizing parameters, in: Proceedings of the 2003 Symposium on Interactive 3D graphics, 2003, pp. 19-26.

[36] N.B. Slonim, L.H. Hamilton, Respiratory Physiology, The C.V. Mosby Company, 1971.

[37] R. Smith, Open dynamics engine, 2003 http://www.q12.org.

[38] D. Stahl, N. Ezquerra, G. Turk, Bag-of-particles as a deformable model, in: IEEE Computer Society: TCGVG, Eurographics Organization, 2002, pp. 141-150.

[39] T. Takahashi, Atlas of the Human Body, Harper Collins, 1994.

[40] J. Teran, S. Blemker, V.N.T. Hing, R. Fedkiw, Finite volume methods for the simulation of skeletal muscle, in: Eurographics/ACM SIGGRAPH Symposium on Computer Animation, 2003, pp. 68-74.

[41] J. Teran, E. Sifakis, S. Blemker, V.N.T. Hing, C. Lau, R. Fedkiw, Creating and simulating skeletal muscle from the visible human data set. IEEE TVCG, 2004. (in press).

[42] D. Terzopoulos, K. Fleischer, Deformable models, Vis. Comput. 4 (6) (1988) 306-331.

[43] D. Terzopoulos, K. Waters, Physically-based facial modelling, analysis, and animation, J. Vis. Comput. Animation 1 (1990) 73-80.

[44] D. Terzopoulos, J. Platt, A. Barr, K. Fleischer, Elastically deformable models, in: Proceedings of SIGGRAPH 87, 1987, pp. 205-214.

[45] Towle, Modern Biology, Holt Rinehart \& Winston, 2000.

[46] X. Tu, D. Terzopoulos, Artificial fishes: physics, locomotion, perception, behavior, in: Proceedings of SIGGRAPH 94, 1994, pp. 43-50.

[47] K. Waters, A muscle model for animating three-dimensional facial expressions, in: Proceedings of SIGGRAPH 87, 1987, 17-24.

[48] J. Wilhelms, A.V. Gelder, Anatomically based modeling, in: Proceedings of SIGGRAPH 1997, 1997, $173-180$.

[49] A. Witkin, W. Welch, Fast animation and control of nonrigid structures, Proc. SIGGRAPH '90 (1990) 243-252.

[50] F. Zajac, Muscle and tendon: properties, models, scaling, and application to biomechanics and motor control, CRC Crit. Rev. Biomed, Eng. 17 (1989) 359-411. 